\title{
Universal scale factors relating mesonic fields and quark operators
}

\author{
Amir H. Faribort* \\ Department of Mathematics/Physics, SUNY Polytechnic Institute, Utica, NY 13502, U.S.A.
}

\author{
J. Hd and T.G. Steel $\bigoplus^{\ddagger}$ \\ Department of Physics and Engineering Physics, \\ University of Saskatchewan, Saskatoon, SK, S7N 5E2, Canada
}

\begin{abstract}
Scale factor matrices relating mesonic fields in chiral Lagrangians and quark-level operators of QCD sum-rules are shown to be constrained by chiral symmetry, resulting in universal scale factors for each chiral nonet. Built upon this interplay between chiral Lagrangians and QCD sum-rules, the scale factors relating the $a_{0}$ isotriplet scalar mesons to their underlying quark composite fields were recently determined. It is shown that the same technique when applied to $K_{0}^{*}$ isodoublet scalars reproduces the same scale factors, confirming the universality property and further validating this connection between chiral Lagrangians and QCD sum-rules which can have nontrivial impacts on our understanding of the low-energy QCD, in general, and the physics of scalar mesons in particular.
\end{abstract}

\section{INTRODUCTION}

In the absence of an exact solution to the strong coupling limit of QCD in terms of fundamental quarks, replacing the fundamental degrees of freedom with light hadrons at low-energies has been shown to be a useful approximation in linear and nonlinear sigma model based approaches. Such approaches have also been proven to be very challenging; nevertheless, the great efforts by many investigators have led to significant progress over the past several decades and resulted in development of important frameworks such as chiral perturbation theory and various effective models [1, 2]. Such frameworks parallel important properties of fundamental QCD by respecting several guiding principles such as chiral symmetry (and its breakdown), $\mathrm{U}(1)_{\mathrm{A}}$ axial anomaly and various assumptions about the QCD vacuum.

However, the quest for understanding the strong interaction phenomena at low-energies based on fundamental QCD has never stopped and important attempts have been made, most important of which is the approach of the lattice QCD program, which despite all the technical challenges has made an enormous progress [1, 37. Still, a framework that can directly connect the low-energy strong interaction data to the fundamental quarks and gluons has not yet emerged. Particularly in the scalar meson sector of low-energy QCD, establishing such a connection is even less trivial. On the experimental side, some of these states are broad and overlap with nearby states, leaving some of their experimental properties vague. On the theoretical side, explaining their mass spectrum and decay properties requires a description beyond the conventional quark-antiquark pattern. For the case of isosinglet scalars, the complexities are significantly greater because these states have the

\footnotetext{
* fariboa@sunypoly.edu

$\dagger$ J.Ho@usask.ca

‡ Tom.Steele@usask.ca
}

same quantum numbers of the QCD vacuum, which can develop a vacuum expectation value and spontaneously break the chiral symmetry. This means that understanding the substructure of isosinglet scalars, which can be composed of not only various two- and four-quark fields but also of glue, is naturally nontrivial and perhaps beyond the current reach of lattice simulations. For a full understanding of scalar sector, it is vital to seek a bridge that can connect the low-energy data all the way to fundamental QCD. Such a solid bridge currently does not exist, and awaits the exact solution to nonperturbative QCD.

In Refs. 8, 9] we demonstrated how a linkage between two existing frameworks, QCD sum-rules [10, 11] (that significantly connect fundamental QCD to hadronic physics through duality relations) and chiral Lagrangians (which are appropriately designed in terms of the hadron fields and can be conveniently used to describe lowenergy data) can provide an approximation of such a bridge. This linkage occurs through scale-factor matrices relating mesonic fields of chiral Lagrangians to quarklevel composite-operator current structures of QCD sumrules. In principle, this connection is quite general and can be established with any formulation of QCD sumrules and any type of chiral Lagrangian, but while the idea is general, one naturally has to make a choice for each of these two frameworks based on their effectiveness in probing a particular channel or process. Since our focus is on scalar channel we use Gaussian sum-rules, a sum-rule methodology designed to handle hadronic mixing [12, 13]; for the chiral Lagrangian side, we use the generalized linear sigma model of [8, 14] which has been applied to a wide range of low-energy scattering and decay channels in which scalar mesons play a dominant role. Specifically, the scale factors were first determined for the isovector scalar sector 8, 9 by connecting the QCD sumrules to the chiral Lagrangian described by the generalized linear sigma model [14]. However, chiral symmetry requires that the scale factors must be universal for all members of the chiral nonets. In the present work (using 
the same framework of Ref. [8, 9]), we demonstrate that the same scale factors are remarkably recovered in the isodoublet scalar sector, providing a crucial test of the universality property.

Establishing universality of these scale factors is essential for exploiting the bridge between chiral Lagrangians and QCD sum-rules to address the long-standing puzzles in the isoscalar sector. The exact relationship between the composite fields of quarks representing a mesonic state (which requires a mass dimension of three or higher), and that of a single mesonic field (of mass dimension one) is not known. We have assumed [8, 9] that this relationship is of a simple form where the underlying composite fields of quarks inside a scalar meson are linearly proportional to the meson field. If this assumption is a good approximation to the exact relationship between the meson fields and their underlying quark fields, then the scale factor adjusting the mass dimensions should reflect certain characteristics of the meson. Chiral symmetry requires that all members of the same chiral nonet have the same scale factor [8, 9] (i.e., the universality condition), which is examined in this work, in testing the proposed bridge, by independently computing the scale factors for the $K_{0}^{*}$ isodoublet scalar system and comparing with the previous computation of these factors for the $a_{0}$ isovector system of Refs. 8, 9].

At the mesonic level, our framework is the generalized linear sigma model of [14] that we use to demonstrate the bridge between chiral Lagrangians and QCD sum-rules. This framework is formulated in terms of a quark-antiquark chiral nonet and a four-quark chiral nonet, and even though there are no direct connections to the underlying quark world, the distinction between two and four-quark nonets is made through the $\mathrm{U}(1)_{\mathrm{A}}$ anomaly. It is shown in [14] (and references therein) how the framework can incorporate various low-energy experimental data to disentangle two- from four-quark components of each members of the scalar meson nonet. While this information is valuable it is not complete. Several four-quark composite fields, each with the same overall quantum numbers of a given scalar meson, can be formed out of different combinations of color and spin (see for example [15]), but these combinations cannot be disentangled solely on the basis of chiral symmetry - a limitation of chiral Lagrangians (such as those of [14]). QCD sumrules, on the other hand, have their own limitations although they directly utilize the specific quark currents, but when probing a complicated scalar meson substructure for which there are numerous possibilities for mixing among two- and four-quark currents, the disentanglement of two- from four-quarks is difficult to achieve in a self contained manner within its framework. Addressing these limitations are examples of the mutual benefits that this bridge provides: The disentanglement of twofrom four-quark currents that can be determined at the mesonic level can enhance (and simplify) the overall analysis of QCD sum-rules, and reciprocally, the self consistency checks within the QCD sum-rules can favor one combination of four-quark currents versus the other and remedy a gap in the chiral Lagrangian approach which, due to the lack of direct connection to the underlying quark fields, is oblivious to various four-quark currents. Establishing an interplay between chiral Lagrangians and QCD sum-rules has been the centerpiece of our proposal in Refs. [8, 9]. This idea is not limited to the scalar channel and/or a specific type of chiral Lagrangian or a particular variant of QCD sum-rules.

\section{METHODOLOGY: SCALE FACTOR MATRICES}

We begin by defining our notation. At the mesonic level, we employ the generalized linear sigma model of 14 which is formulated in terms of two chiral nonets $M$ and $M^{\prime}$ that respectively represent a quark-antiquark nonet and a four-quark nonet (a "molecule" type and/or a diquark-antidiquark type) underlying substructure. Both chiral nonets transform in the same way under chiral transformation but differently under $\mathrm{U}(1)_{\mathrm{A}}$ :

$$
\begin{aligned}
M & \rightarrow U_{L} M U_{R}^{\dagger}, M \rightarrow e^{2 i \nu} M \\
M^{\prime} & \rightarrow U_{L} M^{\prime} U_{R}^{\dagger}, M^{\prime} \rightarrow e^{-4 i \nu} M^{\prime}
\end{aligned}
$$

The axial charge is the main tool for distinguishing these two nonets. Each of these two chiral nonets can be expressed in terms of a scalar and pseudoscalar meson nonet

$$
\begin{aligned}
M & =S+i \phi \\
M^{\prime} & =S^{\prime}+i \phi^{\prime}
\end{aligned}
$$

where the two scalar meson nonets contain the two- and four-quark "bare" (unmixed) scalars

$$
S=\left(\begin{array}{ccc}
S_{1}^{1} & a_{0}^{+} & \kappa^{+} \\
a_{0}^{-} & S_{2}^{2} & \kappa^{0} \\
\kappa^{-} & \bar{\kappa}^{0} & S_{3}^{3}
\end{array}\right), \quad S^{\prime}=\left(\begin{array}{ccc}
{S^{\prime}}_{1}^{1} & a_{0}^{\prime+} & \kappa^{\prime+} \\
a_{0}^{\prime-} & {S^{\prime}}_{2}^{2} & {\kappa^{\prime}}^{0} \\
\kappa^{\prime} & \bar{\kappa}^{\prime 0} & S^{\prime 3}
\end{array}\right)
$$

and similar matrices for $\phi$ and $\phi^{\prime}$. The framework of Ref. [14, 15] provides a detailed analysis of the mixing between these two "bare" nonets and how that results in a description of mass spectrum, decay widths and scattering analysis of scalar as well as pseudoscalar mesons below and above $1 \mathrm{GeV}$. In this picture and of specific interest in this work, the physical isodoublet scalars $K_{0}^{*}(700)$ and $K_{0}^{*}(1430)$ become a linear admixture of two- and four-quark components $\kappa$ and $\kappa^{\prime}$ respectively. Understanding the physical characteristics of $K_{0}^{*}(700)$, particularly its substructure, has posed many challenges and has resulted in numerous investigations [1]. Particularly, the possibility of a non-quark-antiquark nature of this state has been extensively studied [14, 16, 23].

The transformation properties (1) as well as the decompositions (2) are direct consequences of the assumed underlying quark configurations. The two mesonic-level chiral nonets $M$ and $M^{\prime}$ can be mapped to the quark-level 
chiral nonets $M_{\mathrm{QCD}}$ and $M_{\mathrm{QCD}}^{\prime}$. For example, Eq. (1) implies

$$
\left(M_{\mathrm{QCD}}\right)_{a}^{b} \propto\left(\bar{q}_{R}\right)^{b}\left(q_{L}\right)_{a} \Rightarrow\left(S_{\mathrm{QCD}}\right)_{a}^{b} \propto q_{a}(x) \bar{q}^{b}(x),
$$

where $a$ and $b$ are flavor indices and each can take values of 1 to 3 . To make the exact connection to the quark world we need to make a specific choice for the proportionality factor, and with no loss of generality we choose $\left(S_{\mathrm{QCD}}\right)_{a}^{b}=q_{a}(x) \bar{q}^{b}(x)$. The local composite operator $S_{Q C D}$ thus provides a current that is a necessary entity in QCD sum-rule methodology [10]. Similarly, $M_{\mathrm{QCD}}^{\prime}$ can be mapped to quark-level composite field configurations. However, in this case there are several options, each representing a different angular momentum, spin, flavor and color configurations for diquark-antidiquark combination. Here we do not list such quark configurations and the specific form used for our analysis will be given below.

We assume a simple relationship between the quarklevel nonets $M_{\mathrm{QCD}}$ and $M^{\prime}{ }_{\mathrm{QCD}}$ and the physical mesoniclevel nonets $M$ and $M^{\prime}$ via a scale-factor matrix that adjusts the mass dimensions

$$
M=I_{M} M_{\mathrm{QCD}}, M^{\prime}=I_{M^{\prime}} M_{\mathrm{QCD}}^{\prime} .
$$

As shown in [8, 9], chiral symmetry imposes the following constraints on the scale factor matrices

$$
\begin{aligned}
{\left[U_{R}, I_{M}\right] } & =\left[U_{L}, I_{M}\right]=0, \\
{\left[U_{R}, I_{M^{\prime}}\right] } & =\left[U_{L}, I_{M^{\prime}}\right]=0,
\end{aligned}
$$

implying that the scale matrices are multiples of the identity matrix

$$
I_{M}=-\frac{m_{q}}{\Lambda^{3}} \times \mathbb{1}, I_{M^{\prime}}=\frac{1}{\Lambda^{\prime 5}} \times \mathbb{1}
$$

where the (constant) scale factor quantities $\Lambda$ and $\Lambda^{\prime}$ have dimensions of energy that must be determined, and the quark mass factor $m_{q}=\left(m_{u}+m_{d}\right) / 2$ has been chosen to result in renormalization-group invariant currents as discussed below. This methodology can be generalized to include additional substructures (e.g., glueball components) through an additional scale factor. The scale factors $\Lambda$ and $\Lambda^{\prime}$ have been determined in the study of $a_{0}$ isotriplet states [8, 9] and will be redetermined here for isodoublet system to demonstrate universality.

We now consider the specific example of the isodoublets $K_{0}^{*}(700)$ and $K_{0}^{*}(1430)$, for which the physical states are related to the QCD operators via

$$
\mathbf{K}=\left(\begin{array}{c}
K_{0}^{*}(700) \\
K_{0}^{*}(1430)
\end{array}\right)=L_{\kappa}^{-1}\left(\begin{array}{c}
S_{2}^{3} \\
\left(S^{\prime}\right)_{2}^{3}
\end{array}\right)=L_{\kappa}^{-1} I_{\kappa} J^{\mathrm{QCD}}
$$

where $L_{\kappa}^{-1}$ is the rotation matrix that disentangles twofrom four-quark components of isodoublets, $I_{\kappa}$ is formed out of the scale factors defined for the two chiral nonets in (8), and $J^{\mathrm{QCD}}$ is constructed from two- and four-quark operators (the specific form will be given below):

$$
L_{\kappa}^{-1}=\left(\begin{array}{cc}
\cos \theta_{\kappa} & -\sin \theta_{\kappa} \\
\sin \theta_{\kappa} & \cos \theta_{\kappa}
\end{array}\right), I_{\kappa}=\left(\begin{array}{cc}
\frac{-m_{q}}{\Lambda^{3}} & 0 \\
0 & \frac{1}{\Lambda^{\prime 5}}
\end{array}\right) .
$$

Since (9) relates the physical states to QCD operators, we define the projected physical currents $J^{P}=L_{\kappa}^{-1} I_{\kappa} J^{\mathrm{QCD}}$ that define a physical correlation function matrix $\Pi^{P}$ constructed from a physically-projected QCD correlation function matrix $\Pi^{\mathrm{QCD}}$

$$
\begin{gathered}
\Pi^{P}\left(Q^{2}\right)=\widetilde{\mathcal{T}}^{\kappa} \Pi^{\mathrm{QCD}}\left(Q^{2}\right) \mathcal{T}^{\kappa}, \quad \mathcal{T}^{\kappa}=I_{\kappa} L_{\kappa} \\
\Pi_{m n}^{\mathrm{QCD}}(x)=\left\langle 0\left|\mathrm{~T}\left[J_{m}^{\mathrm{QCD}}(x) J_{n}^{\mathrm{QCD}}(0)^{\dagger}\right]\right| 0\right\rangle
\end{gathered}
$$

where $\tilde{\mathcal{T}}^{\kappa}$ denotes the transpose of the matrix $\mathcal{T}^{\kappa}$, and $m$ and $n$ can each take values of 1 (for quark-antiquark current) and 2 (for diquark-antidiquark current) as defined below.

The projected physical correlator matrix is diagonal, providing a self-consistency condition between elements of the QCD correlation function matrix. In our $2 \times 2 K_{0}^{*}$ isodoublet system we have the following constraint from the vanishing of off-diagonal elements. We note that a minor typographical error in Ref. [8] is corrected in (13) and $[9]$.

$$
\Pi_{12}^{\mathrm{QCD}}=-\left[\frac{\widetilde{\mathcal{T}}_{11}^{\kappa} \Pi_{11}^{\mathrm{QCD}} \mathcal{T}_{12}^{\kappa}+\widetilde{\mathcal{T}}_{12}^{\kappa} \Pi_{22}^{\mathrm{QCD}} \mathcal{T}_{22}^{\kappa}}{\widetilde{\mathcal{T}}_{11}^{\kappa} \mathcal{T}_{22}^{\kappa}+\widetilde{\mathcal{T}}_{12}^{\kappa} \mathcal{T}_{12}^{\kappa}}\right]
$$

The relation (13) will be used as input for $\Pi_{12}^{\mathrm{QCD}}$ because the QCD off-diagonal correlator is unknown and not readily calculable because of its complicated higherloop topology.

\section{QCD SUM-RULE ANALYSIS OF SCALE FACTORS}

QCD sum-rule methodologies are based on quarkhadron duality, and apply an integral transform to a dispersion relation relating the QCD and hadronic contributions to the projected physical correlators [10, 11]. The mixing matrix $L_{\kappa}$ must disentangle individual states so a sum-rule method is needed to check whether a residual effect of multiple states is occurring because of an insufficiently accurate mixing matrix. Laplace sum-rules are not suitable because they suppress heavier states, so Gaussian sum-rules will be employed because they provide similar weight to all states [12, 13. The hadronic part of the Gaussian sum-rule is given by

$$
G^{H}(\hat{s}, \tau)=\frac{1}{\sqrt{4 \pi \tau}} \int_{s_{t h}}^{\infty} d t \exp \left[\frac{-(\hat{s}-t)^{2}}{4 \tau}\right] \rho^{H}(t) .
$$

The hadronic spectral function $\rho^{H}$ in 14 is determined from the mesonic fields and a QCD continuum above the 
continuum threshold $s_{0}$ :

$$
\begin{aligned}
\rho^{H}(t) & =\frac{1}{\pi} \operatorname{Im} \Pi^{H}(t)+\theta\left(t-s_{0}\right) \frac{1}{\pi} \operatorname{Im} \Pi^{\mathrm{QCD}}(t) \\
\Pi_{i j}^{H}\left(q^{2}\right) & =\int d^{4} x e^{i q \cdot x}\left\langle 0\left|\mathrm{~T}\left[\mathbf{K}_{i}(x) \mathbf{K}_{j}^{\dagger}(0)\right]\right| 0\right\rangle \\
& =\delta_{i j}\left(\frac{1}{m_{\kappa i}^{2}-q^{2}-i m_{\kappa i} \Gamma_{\kappa i}}\right)
\end{aligned}
$$

The last term in 15 represents the QCD continuum contribution inherent in QCD sum-rule methods [10, 11. The effect of final state interactions in the $\pi K$ channel is quite large near the kappa pole. Within the framework of generalized linear sigma model, these are estimated in 24 .

The hadronic and QCD contributions to the Gaussian sum-rules are now equated:

$$
G^{H}(\hat{s}, \tau)=\widetilde{\mathcal{T}}^{\kappa} G^{\mathrm{QCD}}\left(\hat{s}, \tau, s_{0}\right) \mathcal{T}^{\kappa}
$$

where the QCD continuum has been absorbed from the hadronic side into the QCD contributions. Methods for calculating the QCD prediction $G^{\mathrm{QCD}}$ from the underlying correlation function are reviewed in 13 . The QCD side of $(17)$ is diagonalized via the constraint $(13)$ and the hadronic side of (17) is diagonal because the rotation matrix disentangles the states

$$
G^{H}=\left(\begin{array}{cc}
\left(G^{H}\right)_{11} & 0 \\
0 & \left(G^{H}\right)_{22}
\end{array}\right)=\widetilde{\mathcal{T}}^{\kappa} G^{\mathrm{QCD}} \mathcal{T}^{\kappa}
$$

The resulting diagonal elements of (18) are given by

$$
\begin{gathered}
G_{11}^{H}(\hat{s}, \tau)=a A G_{11}^{\mathrm{QCD}}\left(\hat{s}, \tau, s_{0}^{(1)}\right)-b B G_{22}^{\mathrm{QCD}}\left(\hat{s}, \tau, s_{0}^{(1)}\right) \\
G_{22}^{H}(\hat{s}, \tau)=-a B G_{11}^{\mathrm{QCD}}\left(\hat{s}, \tau, s_{0}^{(2)}\right)+b A G_{22}^{\mathrm{QCD}}\left(\hat{s}, \tau, s_{0}^{(2)}\right) \\
A=\frac{\cos ^{2} \theta_{\kappa}}{\cos ^{2} \theta_{\kappa}-\sin ^{2} \theta_{\kappa}}, B=\frac{\sin ^{2} \theta_{\kappa}}{\cos ^{2} \theta_{\kappa}-\sin ^{2} \theta_{\kappa}} \\
a=\frac{m_{q}^{2}}{\Lambda^{6}}, b=\frac{1}{\left(\Lambda^{\prime}\right)^{10}}
\end{gathered}
$$

where $G_{11}^{H}$ and $G_{22}^{H}$ respectively represent $K_{0}^{*}(700)$ and $K_{0}^{*}(1430)$ contributions, and the factor of $m_{q}^{2}$ is combined with $G_{11}^{Q C D}$ for renormalization-group purposes. Note that each sum-rule has its own continuum threshold represented by $s_{0}^{(1)}$ and $s_{0}^{(2)}$, and the constraint 13 has been used within the QCD prediction.

The scale factors $\Lambda$ and $\Lambda^{\prime}$ for the isodoublet $K_{0}^{*}$ scalar meson system can now be calculated. The QCD currents in 9 are

$$
\begin{gathered}
J^{\mathrm{QCD}}=\left(\begin{array}{c}
J_{1} \\
J_{2}
\end{array}\right), J_{1}=\bar{d} s \\
J_{2}=\sin (\phi) u_{\alpha}^{T} C \gamma_{\mu} \gamma_{5} s_{\beta}\left(\bar{d}_{\alpha} \gamma^{\mu} \gamma_{5} C \bar{u}_{\beta}^{T}-\alpha \leftrightarrow \beta\right) \\
+\cos (\phi) d_{\alpha}^{T} C \gamma_{\mu} s_{\beta}\left(\bar{d}_{\alpha} \gamma^{\mu} C \bar{u}_{\beta}^{T}+\alpha \leftrightarrow \beta\right)
\end{gathered}
$$

where $C$ is the charge conjugation operator and $\cot \phi=$ $1 / \sqrt{2}\left[25\right.$. Given an input of $\cos \theta_{\kappa}=0.4161$ from chiral Lagrangians [14] and the physical mass and width of the $K_{0}^{*}$ states (we use $m_{\kappa}=824 \mathrm{MeV}, \Gamma_{\kappa}=478 \mathrm{MeV}$ for the $K_{0}^{*}(700)$ and $m_{K}=1425 \mathrm{MeV}, \Gamma_{K}=270 \mathrm{MeV}$ for the $K_{0}^{*}(1430)$ to be consistent with [1]) one can solve (19) for the (constant) scale factors $\Lambda$ and $\Lambda^{\prime}$, and optimize the continuum thresholds to minimize the $\hat{s}$ dependence of the scale factors.

The correlation function for the two-quark current $J_{1}$ is given in [26, 27] and the methods of [13] can then be used to form the Gaussian sum-rule:

$$
\begin{gathered}
G_{11}^{\mathrm{QCD}}\left(\hat{s}, \tau, s_{0}\right)= \\
\frac{3}{8 \pi^{2}} \int_{0}^{s_{0}} t d t\left[\left(1+\frac{17}{3} \frac{\alpha_{s}}{\pi}\right)-2 \frac{\alpha_{s}}{\pi} \log \left(\frac{t}{\sqrt{\tau}}\right)\right] W(t, \hat{s}, \tau) \\
+\frac{\pi n_{c} \rho_{c}^{2}}{m_{s}^{*} m_{q}^{*}} \int_{0}^{s_{0}} t J_{1}\left(\rho_{c} \sqrt{t}\right) Y_{1}\left(\rho_{c} \sqrt{t}\right) W(t, \hat{s}, \tau) d t \\
+\exp \left(-\frac{\hat{s}^{2}}{4 \tau}\right)\left[\frac{1}{2 \sqrt{\pi \tau}}\left\langle C_{4}^{s} \mathcal{O}_{4}^{s}\right\rangle-\frac{\hat{s}}{4 \tau \sqrt{\pi \tau}}\left\langle C_{6}^{s} \mathcal{O}_{6}^{s}\right\rangle\right] \\
\quad W(t, \hat{s}, \tau)=\frac{1}{\sqrt{4 \pi \tau}} \exp \left(-\frac{(t-\hat{s})^{2}}{4 \tau}\right) \\
\left\langle C_{4}^{s} \mathcal{O}_{4}^{s}\right\rangle=\left\langle m_{s} \bar{q} q\right\rangle+\frac{1}{2}\left\langle m_{s} \bar{s} s\right\rangle+\frac{1}{8 \pi}\left\langle\alpha_{s} G^{2}\right\rangle \\
\left\langle C_{6}^{s} \mathcal{O}_{6}^{s}\right\rangle=-\frac{1}{2}\left\langle m_{s} \bar{q} \sigma G q\right\rangle-\frac{1}{2}\left\langle m_{q} \bar{s} \sigma G s\right\rangle \\
-\frac{16 \pi}{27} \alpha_{s}\left(\langle\bar{q} q\rangle^{2}+\langle\bar{s} s\rangle^{2}\right)-\frac{48}{9} \alpha_{s}\langle\bar{q} q\rangle\langle\bar{s} s\rangle
\end{gathered}
$$

where $q$ denotes the non-strange $u, d$ quarks and vacuum saturation has been used for the dimension-six (four-quark) condensates. Because $G_{11}^{\mathrm{QCD}}$ is being combined with $m_{q}^{2}$, the combination satisfies a homogenous renormalization-group equation, which requires evaluating all running quantities at the renormalization scale $\nu^{2}=\sqrt{\tau}[13$. Similarly, the Gaussian sum-rule related to the four-quark current $J_{2}$ is obtained via

$$
G_{22}^{\mathrm{QCD}}\left(\hat{s}, \tau, s_{0}\right)=\int_{0}^{s_{0}} d t W(t, \hat{s}, \tau) \rho^{\mathrm{QCD}}(t)
$$

where $\rho^{\mathrm{QCD}}(t)$ is given in [25. Because this result is leading-order, renormalization of the current $J_{2}$ represents a higher-order effect and the Gaussian sum-rule $G_{22}^{\mathrm{QCD}}$ effectively satisfies a homogenous renormalization-group equation, allowing application of the renormalization-group results of Ref. [13]. For the QCD input parameters we use PDG values [1] (quark masses, and $\alpha_{s}$ ) and the following QCD condensate [11, 28, 30] and instanton liquid model parameters [31, 32

$$
\left\langle\alpha_{s} G^{2}\right\rangle=(0.07 \pm 0.02) \mathrm{GeV}^{4},
$$




$$
\begin{gathered}
\frac{\langle\bar{q} \sigma G q\rangle}{\langle\bar{q} q\rangle}=\frac{\langle\bar{s} \sigma G s\rangle}{\langle\bar{s} s\rangle}=(0.8 \pm 0.1) \mathrm{GeV}^{2} \\
\langle\bar{q} q\rangle=-(0.24 \pm 0.2 \mathrm{GeV})^{3},\langle\bar{s} s\rangle=(0.8 \pm 0.1)\langle\bar{q} q\rangle \\
n_{c}=8.0 \times 10^{-4} \mathrm{GeV}^{4}, \rho=1 / 600 \mathrm{MeV} \\
m_{q}^{*}=170 \mathrm{MeV}, m_{s}^{*}=220 \mathrm{MeV}
\end{gathered}
$$

The instanton parameters $\rho$ and $n_{c}$ have an estimated uncertainty of $15 \%$ and the quark zero-mode effective masses $m^{*}$ are correlated with the uncertainty in $\rho$ and the quark condensate [33]. The $m_{s} / m_{q}=27.3$ ratio [1] is of particular importance because it appears in both the QCD inputs and as a parameter in the chiral Lagrangian analysis. The effect of the theoretical uncertainty in the gluon condensate (the dominant QCD condensate effect) on the $a_{0}$ scale factors was examined in Ref. [9] and found to be a small numerical effect. We choose $\tau=3 \mathrm{GeV}^{4}$ consistent with the central value used in Refs. [13, 34.

Fig. 1 shows the $\hat{s}$ dependence of the scale factors for the optimized values of the continuum for both the $a_{0}$ [8, 9] and $K_{0}^{*}$ channels. The $a_{0}$ channel results have been updated from [8, 9] to use $\cos \theta_{a}=0.6304$ consistent with the $m_{s} / m_{q}$ mass ratio used for the $K_{0}^{*}$ analysis. The remarkable independence of the scale factors on the auxiliary sum-rule parameter $\hat{s}$ demonstrates the validity of the scale-factor matrices connecting chiral Lagrangians mesonic fields and the quark-level operators in QCD sum-rules. The small energy $(\hat{s})$ dependence of the scale factors demonstrates that any additional dynamics needed to connect the chiral Lagrangian and QCD sumrule frameworks is small. As is evident from Fig. 1 and Table I, the best-fit predictions of the scale factors clearly demonstrate the crucial universality property required by chiral symmetry.

It is interesting that the scale factors are on the order of magnitude of $\Lambda_{Q C D}$, particularly considering the inclusion of the quark mass factor in (8). Although we cannot make a direct connection between the scale factors and $\Lambda_{Q C D}$, (5) connects mesonic fields with QCD operators and has an implicit connection to the approximate $\Lambda_{Q C D}$ scale of hadronization.

\section{DISCUSSION}

In addition to providing a bridge between chiral Lagrangians and QCD sum-rules, the scale factors can also be related to the chiral Lagrangian vacuum expectations values via $\left\langle S_{1}^{1}\right\rangle=-m_{q}\langle\bar{u} u\rangle / \Lambda^{3}$ and $\left\langle{S^{\prime}}_{1}^{1}\right\rangle \approx$ $1.31\langle\bar{d} d\rangle\langle\bar{s} s\rangle / \Lambda^{\prime 5}$ (note that vacuum saturation effects are embedded in the 1.31 numerical factor). The relation $\left\langle S^{\prime}{ }_{1}^{1}\right\rangle$ is approximate because it depends on the renormalization scale and relies upon the vacuum saturation approximation. As discussed in 8, 9, the resulting agreement is excellent for $\left\langle S_{1}^{1}\right\rangle$ and provides the approximate scale for $\left\langle{S^{\prime}}^{1}\right\rangle$.

In summary, it has been shown that chiral symmetry

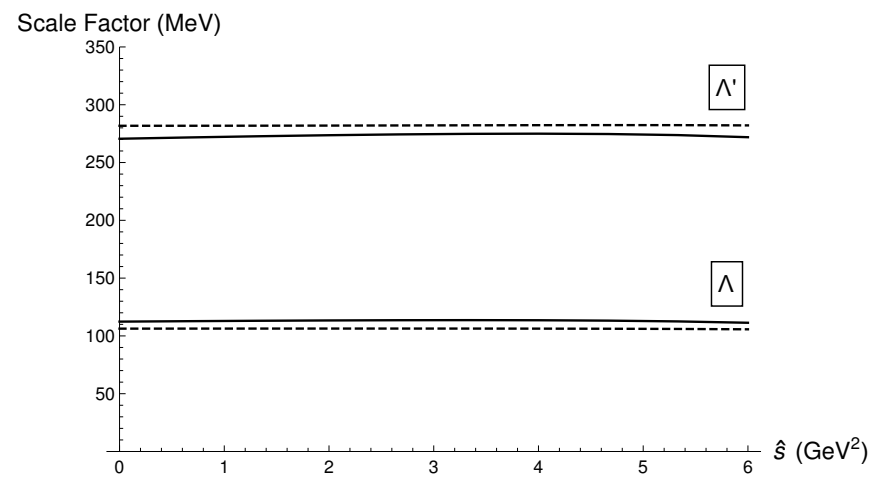

FIG. 1. The scale factors $\Lambda$ (lower pair of curves) and $\Lambda^{\prime}$ (upper pair of curves) are shown as a function of $\hat{s}$ for optimized continuum thresholds in Table I Solid curves are for the $K_{0}^{*}$ channel and dashed curves are for the $a_{0}$ channel.

\begin{tabular}{c|cccc}
\hline Channel & $s_{0}^{(1)}$ & $s_{0}^{(2)}$ & $\Lambda$ & $\Lambda^{\prime}$ \\
\hline$K_{0}^{*}$ & 1.61 & 3.04 & 0.114 & 0.276 \\
$a_{0}$ & 1.68 & 2.88 & 0.106 & 0.282 \\
\hline
\end{tabular}

TABLE I. Values for the optimized scale factors $\Lambda, \Lambda^{\prime}$ and continuum thresholds $s_{0}^{(1)}, s_{0}^{(2)}$ for the $a_{0}$ and $K_{0}^{*}$ channels. All quantities are in appropriate powers of $\mathrm{GeV}$.

transformation properties require that the scale factor matrices serving as a bridge connecting QCD sum-rules and chiral Lagrangians must contain universal scale factors for all sectors of the chiral nonets. The scale factors determined in this work for the $K_{0}^{*}$ system are in remarkable agreement with the corresponding values previously found in the $a_{0}$ channel [ $\underline{6}, 9$, (see Table $\mathrm{I}$ ), providing a key demonstration of the universality property. With evidence for universal scale factors now established, more complicated sectors of the scalar nonets can therefore be simplified by taking input of the universal scale factors from other channels in the nonet. This powerful synergy between chiral Lagrangians and QCD will enable future progress on more challenging and controversial aspects of low-energy hadronic physics. It is also interesting to study application of Eq. (5) in higher spin systems such as in vector and axial-vector meson sectors [35-37] and examine whether quark currents for vectors and axials scale with the same scale factors of Eq. (5) and give rise to the vectors and axials mesonic fields.

\section{ACKNOWLEDGMENTS}

This research was generously supported in part by the SUNY Polytechnic Institute Research Seed Grant Program. TGS is grateful for the hospitality of AHF and SUNY Polytechnic Institute while this work was initiated. TGS and JH are grateful for research funding from the Natural Sciences and Engineering Research Coun- 
cil of Canada (NSERC), and AHF is grateful for a 2019

Seed Grant, and the support of College of Arts and Sci- ences, SUNY Polytechnic Institute. We thank Research Computing at the University of Saskatchewan for computational resources.
[1] M. Tanabashi et al. (Particle Data Group), Phys. Rev. D 98, 030001 (2018).

[2] J.R. Pelaez, Phys. Rept. 658, 1 (2016); E. Klempt and A. Zaitsev, Phys. Rept. 454, 1 (2007).

[3] J. J. Dudek et al. [Hadron Spectrum Collaboration], Phys. Rev. D 88, 094505 (2013).

[4] N. Mathur et al., Phys. Rev. D 76, 114505 (2007).

[5] T. Kunihiro et al. [SCALAR Collaboration], Phys. Rev. D 70, 034504 (2004).

[6] S. Prelovsek, C. Dawson, T. Izubuchi, K. Orginos and A. Soni, Phys. Rev. D 70, 094503 (2004).

[7] C. McNeile et al. [UKQCD Collaboration], Phys. Rev. D 74, 014508 (2006).

[8] A. H. Fariborz, A. Pokraka and T. G. Steele, Mod. Phys. Lett. A 31, 1650023 (2016).

[9] A. H. Fariborz, J. Ho, A. Pokraka and T. G. Steele, arXiv:1909.07243 [hep-ph].

[10] M.A. Shifman, A.I. Vainshtein and V.I. Zakharov, Nucl. Phys. B 147, 385 (1979); M.A. Shifman, A.I. Vainshtein and V.I. Zakharov, Nucl. Phys. B 147, 448 (1979).

[11] L. J. Reinders, H. Rubinstein and S. Yazaki, Phys. Rept. 127, 1 (1985).

[12] R.A. Bertlmann, G. Launer, E. de Rafael, Nucl. Phys. B 250, 61 (1985).

[13] G. Orlandini, T.G. Steele, D. Harnett, Nucl. Phys. A 686, 261 (2001).

[14] A.H. Fariborz, R. Jora and J. Schechter, Phys. Rev. D 72, 034001 (2005); A.H. Fariborz, R. Jora and J. Schechter, Phys. Rev. D 79, 074014 (2009).

[15] A.H. Fariborz, R. Jora and J. Schechter, Phys. Rev. D 77, 094004 (2008).

[16] J.R. Pelaez and A. Rodas, Eur. Phys. J. C 77, 431 (2017); J. R. Pelaez, Phys. Rev. Lett. 92, 102001 (2004).

[17] D. Black, A. H. Fariborz, F. Sannino and J. Schechter, Phys. Rev. D 58, 054012 (1998); Phys. Rev. D 59, 074026
(1999); D. Black, A.H. Fariborz and J. Schechter, Phys. Rev. D 61, 074001 (2000).

[18] J. A. Oller and E. Oset, Phys. Rev. D 60, 074023 (1999).

[19] M. Jamin, J. A. Oller and A. Pich, Nucl. Phys. B 587, 331 (2000).

[20] J. A. Oller, Nucl. Phys. A 727, 353 (2003).

[21] J. A. Oller, Phys. Rev. D 71, 054030 (2005).

[22] Z. H. Guo, J. A. Oller and J. Ruiz de Elvira, Phys. Rev. D 86, 054006 (2012).

[23] F. Giacosa, Phys. Rev. D 75, 054007 (2007).

[24] A.H. Fariborz, E. Pourjafarabadi, S. Zarepour and S.M. Zebarjad, Phys.Rev. D 92, 113002 (2015).

[25] H. X. Chen, A. Hosaka and S. L. Zhu, Phys. Rev. D 76, 094025 (2007); H. X. Chen, A. Hosaka and S. L. Zhu, Phys. Lett. B 650 , 369 (2007).

[26] J. Zhang, H. Y. Jin, Z. F. Zhang, T. G. Steele and D. H. Lu, Phys. Rev. D 79, 114033 (2009).

[27] D. S. Du, J. W. Li and M. Z. Yang, Phys. Lett. B 619, 105 (2005).

[28] S. Narison, Phys. Lett. B 707, 259 (2012).

[29] M. Beneke and H. G. Dosch, Phys. Lett. B 284, 116 (1992).

[30] V. M. Belyaev and B. L. Ioffe, Sov. Phys. JETP 56, 493 (1982).

[31] E. V. Shuryak, Nucl. Phys. B 214, 237 (1983).

[32] T. Schäfer and E. V. Shuryak, Rev. Mod. Phys. 70, 323 (1998).

[33] E. V. Shuryak, Nucl. Phys. B 203, 93 (1982).

[34] D. Harnett and T. G. Steele, Nucl. Phys. A 695, 205 (2001).

[35] G. W. Carter, P. J. Ellis and S. Rudaz, Nucl. Phys. A 603, 367 (1996) Erratum: [Nucl. Phys. A 608, 514 (1996)].

[36] P. Ko and S. Rudaz, Phys. Rev. D 50, 6877 (1994).

[37] D. Parganlija, P. Kovacs, G. Wolf, F. Giacosa and D. H. Rischke, Phys. Rev. D 87, 014011 (2013). 